\title{
EQUICONVERGENCE THEOREMS FOR ORTHONORMAL POLYNOMIALS
}

\author{
G. E. ALBERT AND L. H. MILLER
}

1. Introduction. If $\rho(x)$ is a non-negative function integrable on the finite interval $(a, b)$ and positive on a set of positive measure, there exists a unique set of polynomials $\left\{p_{n}(\rho, x)\right\}$ of degree $n$, $p_{n}(\rho, x)=c_{n} x^{n}+\cdots, c_{n}>0$, which are orthonormal on $(a, b)$ relative to the weight function $\rho(x)$; that is

$$
\int_{a}^{b} \rho(x) p_{n}(\rho, x) p_{m}(\rho, x) d x=\left\{\begin{array}{lll}
0 & \text { if } & n \neq m, \\
1 & \text { if } & m=n .
\end{array}\right.
$$

In the sequel the abbreviation ONP will be used to denote such a set of polynomials.

Given any function $f(x)$ for which the integral $\int_{a}^{b} \rho(x) f(x) d x$ exists, the set of ONP associated with $\rho(x)$ may be used to construct the formal expansion

$$
f(x) \sim \sum_{n=0}^{\infty} a_{n} p_{n}(\rho, x), \quad a_{n}=\int_{a}^{b} \rho(t) f(t) p_{n}(\rho, t) d t .
$$

Much attention has been given to the convergence properties of such series for particular choices of $\rho(x)$. In the succeeding pages known results on this problem are extended by means of what seems to be a new type of proof.

Let $\left\{p_{n}\left(\rho_{1}, x\right)\right\}$ and $\left\{p_{n}\left(\rho_{2}, x\right)\right\}$ be two sets of ONP with different weight functions $\rho_{1}(x)$ and $\rho_{2}(x)$ and let

$$
s_{n}\left(f ; \rho_{i}, x\right)=\sum_{k=0}^{n} a_{k i} p_{k}\left(\rho_{i}, x\right), \quad i=1,2,
$$

denote the partial sums of $n$th degree for the two corresponding expansions (1) associated with $f(x)$. In $\$ \S 5$ and 6 below certain sufficient conditions will be established for the validity of the relation

$$
\lim _{n \rightarrow \infty}\left\{s_{n}\left(f ; \rho_{1}, x\right)-s_{n}\left(f ; \rho_{2}, x\right)\right\}=0 ;
$$

that is, conditions under which the two series mentioned are equiconvergent. It should be stated that the emphasis of the paper is upon method rather than upon specific conditions. The discerning reader

Presented to the Society, April 24, 1943; received by the editors July 9, 1943. 
will easily see arrangements for the weight functions which yield variations of the conditions cited.

The materials from the theory of ONP that are needed in the proofs to follow are quite elementary and are collected for reference in $\$ 2$. Two lemmas are the subject matter of $\$ 3$; from them the remainder of the paper follows essentially as a series of corollaries.

Some interesting relations between bounds for related systems of ONP are indicated in $\$ 4$. These are obtained as by-products of the method of proof for the equiconvergence theorems.

2. Notations and formulas. The symbol $(a, b)$ will denote the closed interval $a \leqq x \leqq b$. It will be assumed without further mention that any weight function and any function $f(x)$ for which a series of type (1) is used will satisfy the conditions set forth for such functions in the introduction. The symbol $L^{2}(\rho ; a, b)$ will denote the class of all measurable functions $f(x)$ for which the integral $\int_{a}^{b} \rho(x)[f(x)]^{2} d x$ exists.

If $f(x)$ and $g(x)$ are in $L^{2}(\rho ; a, b)$, it is well known that

$$
\begin{aligned}
& \int_{a}^{b} \rho(x)[f(x)]^{2} d x=\sum_{k=0}^{\infty} a_{k}^{2}, \\
& \int_{a}^{b} \rho(x) f(x) g(x) d x=\sum_{k=0}^{\infty} a_{k} b_{k}, \quad b_{k}=\int_{a}^{b} \rho(t) g(t) p_{k}(\rho, t) d t,
\end{aligned}
$$

and

$$
\int_{a}^{b} \rho(x)\left[f(x)-s_{n}(f ; \rho, x)\right]^{2} d x \leqq \int_{a}^{b} \rho(x)\left[f(x)-\pi_{n}(x)\right]^{2} d x
$$

for an arbitrary polynomial $\pi_{n}(x)$ of degree $n$.

Also

$$
s_{n}(f ; \rho, x)=\int_{a}^{\delta} \rho(t) f(t) K_{n}(\rho ; x, t) d t
$$

where

$$
\begin{aligned}
K_{n}(\rho ; x, t) & =\sum_{k=0}^{n} p_{k}(\rho, x) p_{k}(\rho, t) \\
& =\frac{c_{n}}{c_{n+1}} \frac{p_{n+1}(\rho, t) p_{n}(\rho, x)-p_{n+1}(\rho, x) p_{n}(\rho, t)}{t-x}
\end{aligned}
$$

In (8), $c_{n}$ is the leading coefficient of $p_{n}(\rho, x)$ and one has

$$
0<c_{n} / c_{n+1} \leqq C=\max \text { of }|a| \text { and }|b| \text {. }
$$


The reader interested in proofs of the above formulas should consult Jackson [1 $]^{1}$ or Szegö [4].

3. Two inequalities. The remaining sections of the paper will be devoted to comparisons of the two sets of $\operatorname{ONP},\left\{p_{n}\left(\rho_{1}, x\right)\right\}$ and $\left\{p_{n}\left(\rho_{2}, x\right)\right\}$, for which the weight functions will be assumed to have the forms

$$
\rho_{1}(x)=\rho(x)\left[\pi_{m}(x)\right]^{2} \text { and } \rho_{2}(x)=\rho(x) \pi_{q}(x) \sigma(x)
$$

or special cases thereof. The following blanket hypotheses will be made concerning the individual factors involved in (10).

(a) The functions $\pi_{m}(x)$ and $\pi_{q}(x)$ are polynomials of degrees $m$ and $q$ respectively and $\rho(x), \pi_{q}(x)$ and $\sigma(x)$ are non-negative on $(a, b)$.

(b) The polynomial $\pi_{q}(x)$ and the function $\sigma(x)$ are such that the product $\pi_{q}(x) \sigma(x)$ is measurable and bounded on $(a, b)$ by a constant $M_{1}$.

(c) The polynomial $\pi_{m}(x)$ and the function $\sigma(x)$ are such that the quotient $\pi_{m}(x) / \sigma(x)$ is measurable and bounded on $(a, b)$ by a constant $M_{2}$.

Lemma 1. Let (a), (b) and (c) be satisfied. If, at a point $\xi$ in $(a, b)$, there exist two positive numbers $\epsilon(\xi)$ and $\gamma(\xi)$ such that when $x$ is in the common part of the intervals $(a, b)$ and $(\xi-\epsilon, \xi+\epsilon)$ the condition

$$
\left|\frac{\pi_{m}(\xi)}{\sigma(\xi)}-\frac{\pi_{m}(x)}{\sigma(x)}\right|<\gamma(\xi) \cdot|x-\xi|
$$

holds, then at the point $\xi$

$$
\left\{\int_{a}^{b} \rho(t) \pi_{q}(t) \sigma(t)\left[\frac{\left\{\pi_{m}(t)\right\}^{2}}{\sigma(t)} K_{n+q}\left(\rho_{1} ; \xi, t\right)-\pi_{q}(\xi) K_{k}\left(\rho_{2} ; \xi, t\right)\right]^{2} d t\right\}^{1 / 2}
$$

$$
\begin{aligned}
\leqq & M_{1}^{1 / 2} M_{2}\left\{\sum_{j=k-m+1}^{n+q}\left[p_{j}\left(\rho_{1}, \xi\right)\right]^{2}\right\}^{1 / 2} \\
& +M_{1}^{1 / 2} C\left\{\left(\frac{4 M_{2}^{2}}{\epsilon^{2}}+\gamma^{2}\right) \sum_{j=k-m}^{k-m+1}\left[p_{j}\left(\rho_{1}, \xi\right)\right]^{2}\right\}^{1 / 2}
\end{aligned}
$$

for all $k$ and $n$ such that $m \leqq k \leqq n$. The constant $C$ is the bound indicated in (9). has

PRoof. Since $\pi_{q}(\xi) p_{j}\left(\rho_{2}, \xi\right)$ is a polynomial of degree $j+q$ in $\xi$, one

1 The numbers in brackets refer to the bibliography. 


$$
\begin{aligned}
\pi_{q}(\xi) p_{j}\left(\rho_{2}, \xi\right) & =\int_{a}^{b} \rho_{1}(t) \pi_{q}(t) p_{j}\left(\rho_{2}, t\right) K_{n+q}\left(\rho_{1} ; \xi, t\right) d t \\
& =\int_{a}^{b} \rho_{2}(t)\left[\frac{\left\{\pi_{m}(t)\right\}^{2}}{\sigma(t)} K_{n+q}\left(\rho_{1} ; \xi, t\right)\right] p_{j}\left(\rho_{2}, t\right) d t
\end{aligned}
$$

for all $j \leqq n$. This implies

$$
\pi_{q}(\xi) K_{k}\left(\rho_{2} ; \xi, t\right) \equiv s_{k}\left(\left[\frac{\pi_{m}^{2}}{\sigma} K_{n+q}\left(\rho_{1}\right)\right] ; \rho_{2}, t\right) .
$$

In view of (6), the left member of (12) is dominated by

$$
\begin{aligned}
\left\{\int_{a}^{b} \rho_{2}(t)\right. & {\left.\left[\frac{\left\{\pi_{m}(t)\right\}^{2}}{\sigma(t)} K_{n+q}\left(\rho_{1} ; \xi, t\right)-\frac{\pi_{m}(\xi) \pi_{m}(t)}{\sigma(\xi)} K_{k-m}\left(\rho_{1} ; \xi, t\right)\right]^{2} d t\right\}^{1 / 2} } \\
\leqq & \left\{M_{1} M_{2}^{2} \int_{a}^{b} \rho_{1}(t)\left[\sum_{j=k-m+1}^{n+q} p_{j}\left(\rho_{1}, \xi\right) p_{j}\left(\rho_{1}, t\right)\right]^{2} d t\right\}^{1 / 2} \\
& +\left\{M_{1} \int_{a}^{b} \rho_{1}(t)\left[\frac{\pi_{m}(t)}{\sigma(t)}-\frac{\pi_{m}(\xi)}{\sigma(\xi)}\right]^{2}\left[K_{k-m}\left(\rho_{1} ; \xi, t\right)\right]^{2} d t\right\}^{1 / 2} .
\end{aligned}
$$

The first term in (14) is clearly bounded by the first term in (12) on the right. In the second term of (14) the range of integration must be broken into the three intervals $(a, \xi-\epsilon),(\xi-\epsilon, \xi+\epsilon)$ and $(\xi+\epsilon, b)$. Using (8), (9), condition (c), and the inequality $|\xi-x| \geqq \epsilon(\xi)$, the contribution to the integral in that term from the first and third subintervals is found to be dominated by

$$
\begin{array}{r}
\frac{4 M_{1} M_{2}^{2} C^{2}}{\epsilon^{2}} \int_{a}^{b} \rho_{1}(t)\left[p_{k-m+1}\left(\rho_{1}, \xi\right) p_{k-m}\left(\rho_{1}, t\right)-p_{k-m+1}\left(\rho_{1}, t\right) p_{k-m}\left(\rho_{1}, \xi\right)\right]^{2} d t \\
\leqq \frac{4 M_{1} M_{2}^{2} C^{2}}{\epsilon^{2}}\left[\left\{p_{k-m}\left(\rho_{1}, \xi\right)\right\}^{2}+\left\{p_{k-m+1}\left(\rho_{1}, \xi\right)\right\}^{2}\right] .
\end{array}
$$

Use of (8), (9) and (11) shows that the contribution to the integral under consideration from $(\xi-\epsilon, \xi+\epsilon)$ is dominated by

$$
M_{1} C^{2} \gamma^{2}\left[\left\{p_{k-m}\left(\rho_{1}, \xi\right)\right\}^{2}+\left\{p_{k-m+1}\left(\rho_{1}, \xi\right)\right\}^{2}\right] \text {. }
$$

Combining all these results one has (12).

The following special case of Lemma 1 is of special importance in the applications.

LEMMA 2. Let the hypotheses (a) and (b) be satisfied. If the quotient $\pi_{m}(x) / \sigma(x)$ satisfies the Lipschitz condition 


$$
\left|\frac{\pi_{m}(x)}{\sigma(x)}-\frac{\pi_{m}(t)}{\sigma(t)}\right|<\lambda|x-t|
$$

for all $x$ and $t$ in $(a, b)$, then, for every point $\xi$ in $(a, b)$,

$$
\begin{gathered}
\left\{\int_{a}^{b} \rho_{2}(t)\left[\frac{\left\{\pi_{m}(t)\right\}^{2}}{\sigma(t)} K_{n+q}\left(\rho_{1} ; \xi, t\right)-\pi_{q}(\xi) K_{k}\left(\rho_{2} ; \xi, t\right)\right]^{2} d t\right\}^{1 / 2} \\
\leqq M_{1}^{1 / 2} M_{2}\left\{\sum_{j=k-m+1}^{n+q}\left[p_{j}\left(\rho_{1}, \xi\right)\right]^{2}\right\}^{1 / 2} \\
+\lambda M_{1}^{1 / 2} C\left\{\sum_{j=k-m}^{k-m+1}\left[p_{j}\left(\rho_{1}, \xi\right)\right]^{2}\right\}^{1 / 2}
\end{gathered}
$$

for all $k$ and $n$ such that $m \leqq k \leqq n$. The constant $C$ is the bound indicated in (9).

Proof. The hypothesis (c) is automatically satisfied. The proof proceeds like that for Lemma 1 except that (8), (9) and the Lipschitz condition are to be applied directly to the second term in (14) to obtain the second term in (16).

4. Bounds for ONP. Lemmas 1 and 2 are powerful tools for establishing bounds for ONP. Since the most interesting bounds are uniform in $x$ on some subset of $(a, b)$, attention will be focused upon the application of Lemma 2; extensions of the results which are obtainable from Lemma 1 are left to the reader.

TheOREM 1. Suppose the hypotheses (a) and (b) and the Lipschitz condition (15) are satisfied. If at a point $\xi$ in $(a, b)$ for which $\pi_{q}(\xi)$ is different from zero there is a number $H_{1}(\xi)$ such that $\left|p_{n}\left(\rho_{1}, \xi\right)\right| \leqq H_{1}$ for all $n$, then there exists a number $H_{2}(\xi)$ such that $\left|p_{n}\left(\rho_{2}, \xi\right)\right| \leqq H_{2}$ for all $n$. Moreover, if for every point of some closed subset of $(a, b)$ on which $\pi_{q}(x)$ is different from zero the bound $H_{1}$ exists and is independent of $x$, then $\mathrm{H}_{2}$ exists and is independent of $x$ on that set.

Proof. Obviously

$$
0 \equiv \int_{a}^{b} \rho_{2}(t) p_{n}\left(\rho_{2}, t\right) \pi_{q}(\xi) K_{n-1}\left(\rho_{2} ; \xi, t\right) d t .
$$

Combining this with (13) for $j=n$, one has

$$
\begin{aligned}
& \pi_{q}(\xi) p_{n}\left(\rho_{2}, \xi\right) \\
& \quad=\int_{a}^{b} \rho_{2}(t) p_{n}\left(\rho_{2}, t\right)\left[\frac{\left\{\pi_{m}(t)\right\}^{2}}{\sigma(t)} K_{n+q}\left(\rho_{1} ; \xi, t\right)-\pi_{q}(\xi) K_{n-1}\left(\rho_{2} ; \xi, t\right)\right] d t .
\end{aligned}
$$


Using Schwarz' inequality, one obtains

$$
\begin{aligned}
&\left|\pi_{q}(\xi) p_{n}\left(\rho_{2}, \xi\right)\right| \leqq\left\{\int_{a}^{b} \rho_{2}(t)\left[p_{n}\left(\rho_{2}, t\right)\right]^{2} d t\right. \\
&\left.\cdot \int_{a}^{b} \rho_{2}(t)\left[\frac{\left\{\pi_{m}(t)\right\}^{2}}{\sigma(t)} K_{n+q}\left(\rho_{1} ; \xi, t\right)-\pi_{q}(\xi) K_{n-1}\left(\rho_{2} ; \xi, t\right)\right]^{2} d t\right\}^{1 / 2} .
\end{aligned}
$$

The first factor on the right is unity; the second factor can be treated by Lemma 2. This gives, for all $n>m$,

$$
\begin{aligned}
\left|\pi_{q}(\xi) p_{n}\left(\rho_{2}, \xi\right)\right| \leqq & M_{1}^{1 / 2} M_{2}\left\{\sum_{j=n-m}^{n+q}\left[p_{j}\left(\rho_{1}, \xi\right)\right]^{2}\right\}^{1 / 2} \\
& +\lambda M_{1}^{1 / 2} C\left\{\sum_{j=n-m-1}^{n-m}\left[p_{j}\left(\rho_{1}, \xi\right)\right]^{2}\right\}^{1 / 2} .
\end{aligned}
$$

The theorem follows since the number of terms on the right is independent of $n$.

Theorem 1 is a generalization of a theorem due to Korous. See Jackson [1, p. 205] or Szegö [4, p. 157]. Results of a similar character have also been established by Peebles [2].

Remark. One or two examples showing the disposition of the factors in (10) may be helpful. Setting $\sigma(x) \equiv \pi_{m}(x) \equiv 1$ in Theorem 1 refers the boundedness of the ONP of weight function $\rho(x) \pi_{q}(x)$ to that of the ONP of weight function $\rho(x)$. This result will be used in $\$ 5$.

The normalized Jacobi polynomials have the weight function $(1-x)^{\alpha}(1+x)^{\beta}$ where $\alpha>-1$ and $\beta>-1$; the interval $(a, b)$ is $(-1,1)$. They can be compared directly with the polynomials $p_{n}(x)=(2 / \pi)^{1 / 2} \cos \theta, x=\cos \theta$, whose weight function is $\left(1-x^{2}\right)^{-1 / 2}$ by the following:

$$
\begin{aligned}
\rho(x) & =(1-x)^{-2 m_{1}-1 / 2}(1+x)^{-2 m_{2}-1 / 2}, \\
\sigma(x) & =(1-x)^{\alpha+2 m_{1}-q_{1}+1 / 2}(1+x)^{\beta+2 m_{2}-q_{2}+1 / 2}, \\
\pi_{q}(x) & =(1-x)^{q_{1}}(1+x)^{q_{2}} \text { and } \pi_{m}(x)=(1-x)^{m_{1}}(1+x)^{m_{2}},
\end{aligned}
$$

where $m_{1}, m_{2}$ are the smallest integers such that $m_{1} \geqq-\alpha / 2-1 / 4$, and $m_{2} \geqq-\beta / 2-1 / 4$ and $q_{1}, q_{2}$ are the smallest integers such that $q_{1} \geqq \alpha+m_{1}+3 / 2$ and $q_{2} \geqq \beta+m_{2}+3 / 2$. Then in Lemma $2, \rho \pi_{q} \sigma$ $=(1-x)^{\alpha}(1+x)^{\beta}$ and $\rho\left[\pi_{m}\right]^{2}=\left(1-x^{2}\right)^{-1 / 2}$ and it is easy to show that the hypotheses are satisfied.

It is fundamental in such applications that the factor $\rho(x)$ need not be integrable for the proofs of Lemmas 1 and 2. One merely makes sure of the integrability of the products $\rho_{1}(x), \rho_{2}(x), \pi_{q}(x) \sigma(x)$, and the quotient $\pi_{m}(x) / \sigma(x)$. 
5. Equiconvergence theorems. Walsh and Wiener [5] have given a necessary and sufficient condition for equiconvergence of two general orthogonal series. Their sufficiency condition will be given below in a form suited to the study of ONP.

LEMMA 3. If $\sigma(x)$ is in $L^{2}(\rho ; a, b)$ and $\left\{p_{n}(\rho, x)\right\}$ and $\left\{p_{n}(\rho \sigma, x)\right\}$ are the ONP with weight functions $\rho(x)$ and $\rho(x) \sigma(x)$, a sufficient condition for

$$
\lim _{n \rightarrow \infty}\left\{s_{n}(f ; \rho, \xi)-s_{n}(f ; \rho \sigma, \xi)\right\}=0, \quad a \leqq \xi \leqq b,
$$

for all $f(x)$ in $L^{2}(\rho ; a, b)$ is that there exist two numbers $N(\xi)$ and $A(\xi)$ such that

$$
\int_{a}^{b} \rho(t)\left[K_{n}(\rho ; \xi, t)-\sigma(t) K_{n}(\rho \sigma ; \xi, t)\right]^{2} d t \leqq A(\xi)
$$

for all $n \geqq N$. Moreover, if $N$ and $A$ are independent of $\xi$ on some subset $S$ of $(a, b)$, then (17) holds uniformly on $S$.

Proof. For an arbitrary point $\xi$ in $(a, b)$ let

$$
\begin{aligned}
a_{k} & =\int_{a}^{b} \rho(t) f(t) p_{k}(\rho, t) d t, \\
b_{k}(\xi) & =\int_{a}^{b} \rho(t)\left[K_{n}(\rho ; \xi, t)-\sigma(t) K_{n}(\rho \sigma ; \xi, t)\right] p_{k}(\rho, t) d t .
\end{aligned}
$$

Now $b_{k}(\xi)=p_{k}(\rho, \xi)-p_{k}(\rho, \xi)=0$ for all $k \leqq n$. Thus by (5)

$$
\begin{aligned}
s_{n}(f ; \rho, \xi)-s_{n}(f ; \rho \sigma, \xi) & =\int_{a}^{b} \rho(t) f(t)\left[K_{n}(\rho ; \xi, t)-\sigma(t) K_{n}(\rho \sigma ; \xi, t)\right] d t \\
& =\sum_{k=n+1}^{\infty} a_{k} b_{k}(\xi) .
\end{aligned}
$$

But then $\left|s_{n}(f ; \rho, \xi)-s_{n}(f ; \rho \sigma, \xi)\right|$ is bounded by

$$
\begin{aligned}
& {\left[\sum_{k=n+1}^{\infty} a_{k}^{2}\right]^{1 / 2}\left[\sum_{k=0}^{\infty}\left\{b_{k}(\xi)\right\}^{2}\right]^{1 / 2}} \\
& \quad \leqq\left[\sum_{k=n+1}^{\infty} a_{k}^{2}\right]^{1 / 2}\left[\int_{a}^{b} \rho(t)\left[K_{n}(\rho ; \xi, t)-\sigma(t) K_{n}(\rho \sigma ; \xi, t)\right]^{2} d t\right]^{1 / 2},
\end{aligned}
$$

where (4) has been used to obtain the inequality. The limit (17) follows at once on using (18) and the well known fact that $\lim _{n \rightarrow \infty} \sum_{k=n+1}^{\infty} a_{k}^{2}=0$. The uniformity clause is evident. 
THEOREM 2. If $\rho(x)$ and $\sigma(x)$ are non-negative and $\sigma(x)$ is bounded and measurable on $(a, b)$ and a polynomial $\pi_{m}(x)$ of degree $m$ can be found such that the quotient $\pi_{m}(x) / \sigma(x)$ satisfies a Lipschitz condition, then (17) holds, for all $f(x)$ in $L^{2}(\rho ; a, b)$, at any point $\xi$ in $(a, b)$ at which $\pi_{m}(\xi)$ is different from zero and $\left|p_{n}(\rho, \xi)\right| \leqq H(\xi)$ where $H(\xi)$ is a number independent of $n$. Moreover, (17) holds uniformly on any closed subset of $(a, b)$ on which $\pi_{m}(x)$ is different from zero and the number $H$ is independent of $x$.

Proof. To demonstrate the inequality (18) set

$$
I_{n}(\xi)=\int_{a}^{b} \rho(t)\left[K_{n}(\rho ; \xi, t)-\sigma(t) K_{n}(\rho \sigma ; \xi, t)\right]^{2} d t .
$$

Add and subtract $\left[\pi_{m}(t)\right]^{2} K_{n}\left(\rho \pi_{m}^{2} ; \xi, t\right)$ inside the bracket in the integrand. Here $K_{n}\left(\rho \pi_{m}^{2} ; \xi, t\right)$ denotes the kernel polynomial (8) for the ONP of weight function $\rho(x)\left[\pi_{m}(x)\right]^{2}$. Applying Minkowski's inequality and a little obvious manipulation, one has

$$
\begin{aligned}
{\left[I_{n}(\xi)\right]^{1 / 2} } & \qquad\left[M_{1} \int_{a}^{b} \rho(t) \sigma(t)\left[\frac{\left\{\pi_{m}(t)\right\}^{2}}{\sigma(t)} K_{n}\left(\rho \pi_{m}^{2} ; \xi, t\right)-K_{n}(\rho \sigma ; \xi, t)\right]^{2} d t\right]^{1 / 2} \\
& +\left[\int_{a}^{b} \rho(t)\left[\left\{\pi_{m}(t)\right\}^{2} K_{n}\left(\rho \pi_{m}^{2} ; \xi, t\right)-K_{n}(\rho ; \xi, t)\right]^{2} d t\right]^{1 / 2},
\end{aligned}
$$

where $M_{1}$ is the bound on $\sigma(x)$. To the first term on the right apply Lemma 2 with $\pi_{q}(x) \equiv 1, k=n$; to the second term apply Lemma 2 with $\pi_{q}(x) \equiv \sigma(x) \equiv 1$ and $k=n$. The result is

$$
\begin{aligned}
{\left[I_{n}(\xi)\right]^{1 / 2} \leqq } & \left(M_{1} M_{2}+M_{3}\right)\left[\sum_{j=n-m+1}^{n}\left\{p_{j}\left(\rho \pi_{m}^{2}, \xi\right)\right\}^{2}\right]^{1 / 2} \\
& +\lambda C\left(M_{1}+1\right)\left[\sum_{j=n-m}^{n-m+1}\left\{p_{j}\left(\rho \pi_{m}^{2}, \xi\right)\right\}^{2}\right]^{1 / 2}
\end{aligned}
$$

for all $n \geqq m$. The constants $M_{2}$ and $M_{3}$ are bounds on $\pi_{m} / \sigma$ and $\pi_{m}$. By the remark following Theorem 1 the system $\left\{p_{n}\left(\rho \pi_{m}^{2}, \xi\right)\right\}$ is bounded as to $n$ at any point $\xi$ such that $\pi_{m}(\xi)$ is different from zero and at which the system $\left\{p_{n}(\rho, \xi)\right\}$ is so bounded. This proves the inequality (18) under the stated hypotheses. The uniformity clause is evident.

The following more general theorem is obtained by applying Lemma 1 to the integral $I_{n}(\xi)$. 
THEOREM 3. Let $\rho(x)$ and $\sigma(x)$ be non-negative and $\sigma(x)$ be bounded and measurable on $(a, b)$. Let $\xi$ be a point in $(a, b)$. If there is a polynomial $\pi_{m}(x)$ of degree $m$ such that $\pi_{m}(\xi)$ is different from zero and $\pi_{m}(x) / \sigma(x)$ satisfies the condition (11) of Lemma 1 and if there is a number $H(\xi)$ independent of $n$ such that $\left|p_{n}(\rho, \xi)\right| \leqq H(\xi)$ for all $n$, then (17) holds for all $f(x)$ in $L^{2}(\rho ; a, b)$.

Theorems 2 and 3 furnish no information about equiconvergence at a point where the auxiliary polynomial $\pi_{m}(x)$ vanishes. It is not difficult to construct examples which show that such equiconvergence cannot be expected for all functions in $L^{2}(\rho ; a, b)$.

6. Applications. Let $(a, b)$ be $(-1,1)$ and let $\rho(x)$ and $\sigma(x)$ have the forms

$$
\rho(x)=\left(1-x^{2}\right)^{-1 / 2} \text { and } \sigma(x)=\tau(x) \prod_{k=1}^{h}\left|x-x_{k}\right|^{\lambda_{k}}
$$

where $-1 \leqq x_{1}<x_{2}<\cdots<x_{h} \leqq 1 ; \lambda_{k} \geqq 0, k=1,2, \cdots, h$, and $0<A$ $\leqq \tau(x) \leqq B$. Theorem 2 applies if $\tau(x)$ satisfies a Lipschitz condition on $(-1,1)$ and Theorem 3 applies at any point $\xi$ in $(-1,1)$ distinct from all the points $x_{k}, k=1,2, \cdots, h$, provided that there is a pair of numbers $\lambda(\xi)$ and $\epsilon(\xi, \lambda)$ such that

$$
|\tau(x)-\tau(\xi)|<\lambda|x-\xi|,
$$

for all $x$ in $(-1,1)$ such that $|x-\xi|<\epsilon$. In either case the polynomial $\pi_{m}(x)$ is chosen to be $\prod_{k=1}^{h}\left(x-x_{k}\right)^{m_{k}}$, where $m_{k}$ is the smallest integer such that $m_{k}-\lambda_{k} \geqq 1$. These results compare the convergence of the series (1) for the ONP of weight function $\rho(x) \sigma(x)$ with the convergence of the Fourier cosine series on the open intervals $x_{i}<x<x_{i+1}$, $i=1,2, \cdots, h$. Either case includes the Jacobi polynomials of weight function $(1-x)^{\alpha}(1+x)^{\beta}$ where $\alpha, \beta>-1 / 2$. The cases where $\alpha$ or $\beta$ or both are less than $-1 / 2$ are also easily handled. For example, if $\alpha=\beta=-3 / 4$, set $\rho(x)=\left(1-x^{2}\right)^{-8 / 4}$ and $\sigma(x)=\left(1-x^{2}\right)^{1 / 4}$ and choose $\pi_{m}(x)$ appropriately.

The case above using Theorem 3 essentially includes a theorem of Szegö [4; Theorem 13.1.2, p. 307]. The case using Theorem 2 is comparable to results of Peebles [2]. The methods of the present paper are certainly more elementary and more direct than those used by either of the above authors.

\section{BIBLIOGRAPHY}

1. D. Jackson, Fourier series and orthogonal polynomials, 1941. 
2. G. H. Peebles, An equivalence theorem for series of orthogonal polynomials, Proc. Nat. Acad. Sci. U.S.A. vol. 25 (1939) p. 97.

3. - On equivalence of certain types of series of orthonormal functions, Bull. Amer. Math. Soc. vol. 48 (1942) pp. 556-561.

4. G. Szegö, Orthogonal polynomials, New York, 1939.

5. J. L. Walsh and N. Wiener, The equivalence of expansions in terms of orthogonal functions, Journal of Mathematics and Physics, Massachusetts Institute of Technology vol. 1 (1922) p. 103.

Ohio State University 